TRANSACTIONS OF THE

AMERICAN MATHEMATICAL SOCIETY

Volume 352, Number 6, Pages 2689-2700

S 0002-9947(00)02485-5

Article electronically published on February 24, 2000

\title{
GROUP ACTIONS AND GROUP EXTENSIONS
}

\author{
ERGÜN YALÇIN
}

\begin{abstract}
In this paper we study finite group extensions represented by special cohomology classes. As an application, we obtain some restrictions on finite groups which can act freely on a product of spheres or on a product of real projective spaces. In particular, we prove that if $(Z / p)^{r}$ acts freely on $\left(S^{1}\right)^{k}$, then $r \leq k$.
\end{abstract}

\section{INTRODUCTION}

Let $G$ be a finite group. An extension of $G$ by an abelian group $M$,

$$
0 \rightarrow M \rightarrow \Gamma \rightarrow G \rightarrow 1
$$

is called special if its restrictions to cyclic subgroups of $G$ do not split. Extensions of this kind arise naturally in many contexts including group theory, classification of flat manifolds [13], and group actions on special manifolds [3]. In this paper we establish inequalities for such extensions, and then look at the free group actions on the $k$-torus and products of real projective spaces. In both of these cases short exact homotopy sequences for corresponding coverings are special extensions. As an application, we solve special cases of some well known problems.

We first consider extensions with $M=\mathbf{Z}^{k}$, a free abelian group of rank $k$. In this case a group extension $0 \rightarrow \mathbf{Z}^{k} \rightarrow \Gamma \rightarrow G \rightarrow 1$ is special if and only if $\Gamma$ is torsion free. Our main result is the following :

Theorem 3.2. Let $G$ be a finite group, and let $0 \rightarrow \mathbf{Z}^{k} \rightarrow \Gamma \rightarrow G \rightarrow 1$ be an extension of $G$. If $\Gamma$ is torsion free, then $r_{p}(G) \leq k$ for all $p|| G \mid$.

Here $r_{p}(G)$ denotes the $p$-rank of $G$, the largest integer $r$ such that $(\mathbf{Z} / p)^{r} \subseteq G$. As an application, we have

Corollary 5.2. If $G=(\mathbf{Z} / 2)^{r}$ acts freely on $X \simeq\left(S^{1}\right)^{k}$, then $r \leq k$.

The existence of this inequality for free $(\mathbf{Z} / p)^{r}$ actions on $\left(S^{n}\right)^{k}$ was stated as a problem by P.E. Conner after P.A. Smith's results on finite group actions on spheres. Under the assumption of trivial action on homology, this problem was solved by G. Carlsson [7], 8]. Later W. Browder [5] by using exponents, and Benson and Carlson [4] by using machinery of varieties for modules, gave alternative proofs of this result. The homologically nontrivial case involved some module theoretical difficulties overcome by Adem and Browder in [2]. They obtained the inequality

Received by the editors January 30, 1998.

1991 Mathematics Subject Classification. Primary 57S25; Secondary 20J06, 20C15.

Key words and phrases. Group extensions, special classes, products of spheres, cohomology of groups.

Partially supported by NATO grants of the Scientific and Technical Research Council of Turkey. 
$r \leq k$ except in the cases $p=2$ and $n=1,3$, or 7 . Corollary 5.2 solves the $p=2$ and $n=1$ cases, leaving the other two cases open.

Adem and Browder also questioned whether or not the stronger inequality

$$
r \leq \operatorname{dim}_{\mathbf{F}_{p}} H_{n}\left(X, \mathbf{F}_{p}\right)^{G}
$$

holds 2. Later Adem and Benson established this inequality for permutation modules [1. In section 5, we give a different proof of this result for $n=1$.

We then look at the central extensions $\quad 0 \rightarrow M \rightarrow \Gamma \rightarrow G \rightarrow 1$ where $G$ is a finite group, $M$ is a free abelian group, and $G$ acts on $M$ trivially. We show that if such an extension is special, then $G$ must be abelian. As a topological application, we conclude that nonabelian groups cannot act freely on the $k$-torus $\left(S^{1}\right)^{k}$ with a trivial action on homology.

Next, we consider the central extensions

$$
0 \rightarrow E \rightarrow Q \rightarrow G \rightarrow 1
$$

where both $E$ and $G$ are elementary abelian 2-groups of rank $k$ and $r$ respectively. Observe that these extensions are special if and only if $r_{2}(Q)=k$. In this case every element of order 2 in $Q$ is central, which is known as the $2 \mathrm{C}$ condition for $Q$. For special central extensions as (1), Cusick showed that the inequality $r \leq 2 k$ must hold [9]. We first prove a refinement of this result which gives a stronger inequality

$$
r \leq k+\operatorname{rk} Q^{\prime}
$$

where $Q^{\prime}$ is the commutator subgroup of $Q$. Then in Section 7 we obtain

$$
\operatorname{rk} Q^{\prime}=\operatorname{dim}_{\mathbf{F}_{p}}\left(\operatorname{im} d_{3}\right)
$$

where $d_{3}: H^{2}(E, \mathbf{Z}) \rightarrow H^{3}(G, \mathbf{Z})$ is a differential on the Hochschild-Serre spectral sequence $H^{*}\left(G, H^{*}(E, \mathbf{Z})\right) \Rightarrow H^{*}(Q, \mathbf{Z})$ for (1). Applying these to the group extensions associated to the group actions on products of real projective spaces, we obtain the following:

Theorem 8.3. If $G=(\mathbf{Z} / 2)^{r}$ acts freely and mod 2 homologically trivially on a finite complex $X \simeq \prod_{i=1}^{k} \mathbf{R} P^{2 m_{i}+1}$ where $m_{i}>0$ for all $i$, then $r \leq \mu\left(m_{1}\right)+\cdots+$ $\mu\left(m_{k}\right)$, where $\mu\left(m_{i}\right)=1$ for $m_{i}$ even and $\mu\left(m_{i}\right)=2$ for $m_{i}$ odd.

This solves a special case of a conjecture on elementary abelian 2-group actions on products of projective spaces, stated by L.W. Cusick $[9$.

Now, we fix some notation. Throughout this paper $Z(G)$ will denote the center of the group $G$, and $G^{\prime}$ will be the commutator subgroup of $G$. Also, for a prime number $p, \mathbf{Z} / p$ will be the integers $\bmod p$, while $\mathbf{F}_{p}$ will denote the field of $p$ elements.

The results in this paper form part of a doctoral dissertation, written at the University of Wisconsin. I am indebted to my thesis advisor Alejandro Adem for his guidance and constant support. I also would like to thank Nobuaki Yagita for pointing out a mistake in an earlier version of this paper.

\section{Rational Representations of $(\mathbf{Z} / 2)^{r}$}

Let $G$ be an elementary abelian 2-group of rank $r$. If $\pi: G \rightarrow \mathbf{Z} / 2$ is a surjection, then the composition $G \rightarrow \mathbf{Z} / 2 \rightarrow \mathbf{Q}(-1)^{*}$ determines an irreducible $\mathbf{Q} G$-module which will be denoted by $M(\pi)$. In fact, all nontrivial irreducible representations 
arise in this way (see 2]) and therefore, any $\mathbf{Q} G$-module $M$ can be expressed uniquely as

$$
M \cong \mathbf{Q}^{m} \oplus\left(\bigoplus_{i=1}^{t} M\left(\pi_{i}\right)\right) .
$$

For a $\mathbf{Z} G$-module $L$, let $\mathbf{Q} L$ denote the corresponding rational representation $\mathbf{Q} \otimes \mathbf{z} L$.

Lemma 2.1. Let $L$ be a $\mathbf{Z}$ free $\mathbf{Z} G$-module such that $\mathbf{Q} L$ is a simple $\mathbf{Q} G$-module. Then, for every $\alpha \in H^{2}(G, L)$ there is an index 2 subgroup $H \subseteq G$ such that $\operatorname{res}_{H}^{G} \alpha=0$.

Proof. We first show that when $Q L$ is an irreducible module, $H^{2}(G, L)$ has exponent 2. This is clear when $Q L \cong Q$ because then, $L \cong \mathbf{Z}$ and $H^{2}(G, \mathbf{Z}) \cong(\mathbf{Z} / 2)^{r}$. When $\mathbf{Q} L$ is nontrivial, by the above classification, it is isomorphic to $M(\pi)$ for some $\pi: G \rightarrow \mathbf{Z} / 2$. Let $K=\operatorname{ker} \pi$ and let $C$ be a cyclic subgroup of $G$ such that $G \cong K \times C$. The exact sequence $C \rightarrow G \rightarrow K$ gives a Lyndon-Hochschild-Serre spectral sequence $H^{*}\left(K, H^{*}(C, L)\right) \Rightarrow H^{*}(G, L)$ where even dimensional horizontal lines vanish because $L^{C}=0$. Since $L$ is one dimensional and $C$ acts on $L$ by multiplication by $(-1), H^{1}(C, L) \cong L / 2 L \cong \mathbf{Z} / 2$, which gives that $H^{2}(G, L) \cong$ $H^{1}\left(K, \mathbf{F}_{2}\right)$ has exponent 2 .

Now, consider the following long exact sequence

$$
\rightarrow H^{1}(G, L / 2 L) \longrightarrow H^{2}(G, L) \stackrel{\times 2}{\longrightarrow} H^{2}(G, L) \longrightarrow H^{2}(G, L / 2 L) \rightarrow
$$

which comes from the coefficient sequence $0 \rightarrow L \stackrel{\times 2}{\longrightarrow} L \longrightarrow L / 2 L \rightarrow 0$. Since multiplication by 2 is zero on $H^{2}(G, L)$, there is a class $\alpha^{\prime} \in H^{1}(G, L / 2 L)$ which maps to $\alpha$. But, by $L / 2 L \cong \mathbf{F}_{2}$, and hence $H^{1}(G, L / 2 L) \cong \operatorname{Hom}\left(G, \mathbf{F}_{2}\right)$. Let $H \subseteq G$ be the kernel of the map that corresponds to $\alpha^{\prime}$. Then $\operatorname{res}_{H}^{G} \alpha^{\prime}=0$, and hence $\operatorname{res}_{H}^{G} \alpha=0$.

By using an induction on simple summands of $\mathbf{Q} M$ we get

Lemma 2.2. Let $M$ be a $\mathbf{Z}$ free $\mathbf{Z} G$-module with $\operatorname{rk} M<r$. Then, for any $\alpha \in$ $H^{2}(G, M)$ there exists a nontrivial subgroup $H \subseteq G$ such that $\operatorname{rk} H=(r-\operatorname{rk} M)$ and $\operatorname{res}_{H}^{G} \alpha=0$.

Proof. (By induction on $\operatorname{rk} M$.)

When $\mathbf{Q} M$ is irreducible, this lemma follows from Lemma 2.1 Assume $\mathbf{Q} M$ is not irreducible, then $\mathbf{Q} M \cong S \oplus U$ for some nonzero $\mathbf{Q} G$-modules $S$ and $U$. Let $q$ be the projection map from $\mathbf{Q} M$ to $U$. Set $N=q(M)$ and $L=\operatorname{ker} q \cap M$. Then we have a short exact sequence of $\mathbf{Z} G$ lattices

$$
0 \rightarrow L \stackrel{i}{\longrightarrow} M \stackrel{q}{\longrightarrow} N \rightarrow 0
$$

where $\mathbf{Q} L \cong S$ and $\mathbf{Q} N \cong U$. Consider the corresponding long exact sequence for cohomology

$$
\rightarrow H^{2}(G, L) \stackrel{i^{*}}{\longrightarrow} H^{2}(G, M) \stackrel{q^{*}}{\longrightarrow} H^{2}(G, N) \rightarrow .
$$

Let $\alpha$ be any element in $H^{2}(G, M)$. Then $q^{*}(\alpha) \in H^{2}(G, N)$ and by induction there is a subgroup $K$ in $G$ such that $\operatorname{rk} K=r-\operatorname{rk} N$ and $r e s_{K}^{G} q^{*}(\alpha)$ is zero. From the 
commutative diagram

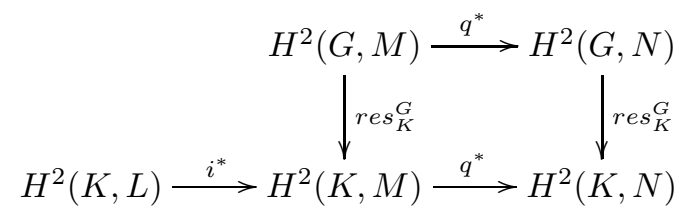

we get $q^{*}\left(\operatorname{res}_{K}^{G} \alpha\right)=0$. Hence there exists an element in $H^{2}(K, L)$, say $\alpha^{\prime}$, which maps to $\operatorname{res}_{K}^{G} \alpha$. By induction again, there is a subgroup $H \subseteq K$ such that $\operatorname{rk} H=\operatorname{rk} K-\operatorname{rk} L$ and $r e s_{H}^{K} \alpha^{\prime}$ is zero. Then

$$
\operatorname{res}_{H}^{G} \alpha=\operatorname{res}_{H}^{K} \operatorname{res}_{K}^{G} \alpha=\operatorname{res}_{H}^{K} i^{*}\left(\alpha^{\prime}\right)=i^{*}\left(\operatorname{res}_{H}^{K} \alpha^{\prime}\right)=0
$$

and

$$
\operatorname{rk} H=r-\operatorname{rk} N-\operatorname{rk} L=r-\operatorname{rk} M .
$$

So, $H$ is a subgroup as desired.

\section{THE MAIN THEOREM}

First, we prove the following:

Lemma 3.1. Let $G=(\mathbf{Z} / p)^{r}$, and let $M$ be a $\mathbf{Z}$ free $\mathbf{Z} G$-module. If $0 \rightarrow M \rightarrow$ $\Gamma \rightarrow G \rightarrow 1$ is an extension such that $\Gamma$ is torsion free, then

$$
\text { rk } H \leq \operatorname{dim}_{\mathbf{F}_{p}}\left(\mathbf{F}_{p} \otimes M\right)^{G}
$$

where $H \subseteq G$ is the subgroup of elements in $G$ acting trivially on $M$.

Proof. Let us fix a set of representatives in $\Gamma$ for elements of $G$, and let us denote the representative of an element $g \in G$ by $\hat{g}$. Let $\psi: H \rightarrow M / p M$ be the $p^{t h}$ power $\operatorname{map}$ in $\bmod p$, i.e. $\psi(h)=(\hat{h})^{p}(\bmod p)$. We will show that $\psi$ is an injection into $(M / p M)^{G}$ which will imply the desired inequality.

First observe that for $h, k \in H,\left[\hat{h},(\hat{k})^{p}\right]=0$ because $(\hat{k})^{p}$ is in $M$ and $h$ acts on $M$ trivially. Then note that

$$
0=\left[\hat{h},(\hat{k})^{p}\right]=[\hat{h}, \hat{k}]+[\hat{h}, \hat{k}]^{\hat{k}}+\cdots+[\hat{h}, \hat{k}]^{(\hat{k})^{p-1}}=p[\hat{h}, \hat{k}] .
$$

Hence $[\hat{h}, \hat{k}]=0$, because $[\hat{h}, \hat{k}]$ is in $M$ and $M$ is $\mathbf{Z}$-free. From this it follows that $\psi$ is a homomorphism, and the injectivity follows from the fact that $\Gamma$ is torsion free.

Finally, we compute $(g-1) \psi(h)$ as

$$
\left[\hat{g},(\hat{h})^{p}\right]=[\hat{g}, \hat{h}]+[\hat{g}, \hat{h}]^{\hat{h}}+\cdots+[\hat{g}, \hat{h}]^{(\hat{h})^{p-1}}=p[\hat{g}, \hat{h}] \equiv 0 \quad(\bmod p) .
$$

Hence the image of $\psi$ is in $(M / p M)^{G}$. This completes the proof.

Now, we state our main result.

Theorem 3.2. Let $G$ be a finite group, and let $0 \rightarrow \mathbf{Z}^{k} \rightarrow \Gamma \rightarrow G \rightarrow 1$ be an extension of $G$. If $\Gamma$ is torsion free, then $r_{p}(G) \leq k$ for all $p|| G \mid$.

Proof. Observe that it is enough to prove the theorem for $G=(\mathbf{Z} / p)^{r}$. Let $M=\mathbf{Z}^{k}$, and let $\alpha \in H^{2}(G, M)$ be the extension class for $0 \rightarrow M \rightarrow \Gamma \rightarrow G \rightarrow 1$ as explained in Chapter IV of [6]. We use different arguments for the $p=2$ and $p>2$ cases.

$p=2$ case. Assume $r>k$, then by Lemma 2.2 there exists a nontrivial subgroup $H \subseteq G$ such that $\operatorname{res}_{H}^{G} \alpha=0$ which implies that $\pi^{-1}(H) \rightarrow H$ splits, where 
$\pi: \Gamma \rightarrow G$. Thus there is a subgroup $K \subseteq \pi^{-1}(H) \subseteq \Gamma$ that is isomorphic to $H$. Since $H$ is a torsion group, $\Gamma$ cannot be torsion free.

$p>2$ case. Let $H \subseteq G$ be the subgroup of elements that act trivially on $M=\mathbf{Z}^{k}$. Then, by Lemma 3.1

$$
\operatorname{rk} H \leq \operatorname{dim}_{\mathbf{F}_{p}}\left(\mathbf{F}_{p} \otimes M\right)^{G} .
$$

On the other hand, $G / H$ acts faithfully on $M$, and for faithful modules we have

Theorem 3.3 (Adem and Browder [2]). Let $K$ be an elementary abelian p-group with $p>2$ and $M$ be a $\mathbf{Z}$ free $\mathbf{Z} K$-module. If $M$ is a faithful representation, then

$$
\operatorname{rk} K \leq \frac{1}{p-2}\left(\operatorname{dim}_{\mathbf{F}_{p}} \mathbf{F}_{p} \otimes M-\operatorname{dim}_{\mathbf{F}_{p}}\left(\mathbf{F}_{p} \otimes M\right)^{K}\right) .
$$

This immediately gives an equality for $G / H$ which, together with (2), implies that

$$
r \leq \operatorname{dim}_{\mathbf{F}_{p}}\left(\mathbf{F}_{p} \otimes M\right)^{G}+\frac{1}{p-2}\left(\operatorname{dim}_{\mathbf{F}_{p}} \mathbf{F}_{p} \otimes M-\operatorname{dim}_{\mathbf{F}_{p}}\left(\mathbf{F}_{p} \otimes M\right)^{G}\right) \leq k .
$$

\section{Permutation module case}

In this section we show that the result of Section 3 can be strengthened in the case of a permutation module. We start with a definition.

Definition 4.1. Let $G$ be a finite group, and let $M$ be a $\mathbf{Z} G$-module. A class $\alpha \in H^{n}(G, M)$ is called special if it has nonzero restrictions to every cyclic subgroup of $G$.

So, an extension $0 \rightarrow M \rightarrow \Gamma \rightarrow G \rightarrow 1$ is special if and only if the corresponding class in $H^{2}(G, M)$ is a special class.

Lemma 4.2. If $\alpha \in H^{2}(G, M)$ is a special class, then its image under the mod $p$ reduction map $H^{2}(G, M) \rightarrow H^{2}\left(G, \mathbf{F}_{p} \otimes M\right)$ is also special.

Proof. Let $\alpha \in H^{2}(G, M)$ be the class representing the extension $\quad 0 \rightarrow M \rightarrow \Gamma \rightarrow$ $G \rightarrow 1$. For a cyclic subgroup $C \subseteq G$, consider the following commutative diagram

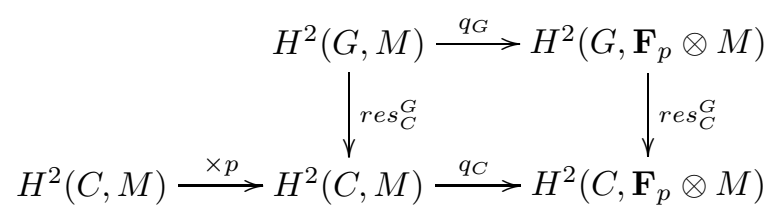

where the rows come from the coefficient sequence $0 \rightarrow M \stackrel{\times p}{\longrightarrow} M \rightarrow \mathbf{F}_{p} \otimes M \rightarrow 0$. Since multiplication by $|C|$ on $H^{2}(C, M)$ is zero, the map $q_{C}$ is injective. Hence, the fact that $\operatorname{res}_{C}^{G} \alpha \neq 0$ implies that

$$
\operatorname{res}_{C}^{G} q_{G}(\alpha)=q_{C}\left(\operatorname{res}_{C}^{G} \alpha\right) \neq 0 .
$$

Since this is true for all cyclic subgroups $C \subseteq G, q_{G}(\alpha)$ is special.

Proposition 4.3. Let $0 \rightarrow M \rightarrow \Gamma \rightarrow G \rightarrow 1$ be a special extension, where $G=(\mathbf{Z} / p)^{r}$ and $M=\mathbf{Z}^{k}$. Suppose $\mathbf{F}_{p} \otimes M$ is a permutation $\mathbf{F}_{p} G$-module. Then

$$
\operatorname{rk} G \leq \operatorname{dim}_{\mathbf{F}_{p}}\left(\mathbf{F}_{p} \otimes M\right)^{G} .
$$


Proof. Let $0 \rightarrow M \rightarrow \Gamma \rightarrow G \rightarrow 1$ be a special extension represented by the class $\alpha^{\prime} \in H^{2}(G, M)$. Let $\alpha$ be its image under the $\bmod p$ reduction map $H^{2}(G, M) \rightarrow$ $H^{2}\left(G, \mathbf{F}_{p} \otimes M\right)$. By the above lemma, $\alpha$ is also special. Now, if $\mathbf{F}_{p} \otimes M$ is a permutation module, then it is of the form $\mathbf{F}_{p} \otimes M=\bigoplus_{i=1}^{l} \mathbf{F}_{p}\left[G / H_{i}\right]$ for some collection of subgroups $H_{i} \subseteq G$. Let $\pi_{i}: G \rightarrow H_{i}, 1 \leq i \leq l$, be a set of fixed projections. For each $i$, consider the composition

$$
\phi_{i}: H^{2}\left(G, \mathbf{F}_{p}\left[G / H_{i}\right]\right) \stackrel{\psi_{i}}{\longrightarrow} H^{2}\left(H_{i}, \mathbf{F}_{p}\right) \stackrel{\pi_{i}^{*}}{\longrightarrow} H^{2}\left(G, \mathbf{F}_{p}\right)
$$

where the first map is the isomorphism in "Shapiro's lemma" (see page 73 of [5]) and the second map induces from $\pi_{i}: G \rightarrow H_{i}$.

Let $\theta=\left(\phi_{1}\left(\alpha_{1}\right), \ldots, \phi_{l}\left(\alpha_{l}\right)\right)$. We claim that $\theta \in H^{2}\left(G, \bigoplus_{i=1}^{l} \mathbf{F}_{p}\right)$ is a special class. To prove the claim, it is enough to show that for every cyclic subgroup $C \subseteq G$, there is an $i$ such that $\operatorname{res}_{C}^{G} \phi_{i}\left(\alpha_{i}\right) \neq 0$. Take a cyclic subgroup $C \subseteq G$. Since $\alpha$ is special, $r e s_{C}^{G} \alpha_{i} \neq 0$ for some $i$. If $C \nsubseteq H_{i}$, then $\operatorname{res}_{C}^{G} \mathbf{F}_{p}\left[G / H_{i}\right]$ is a free $\mathbf{F}_{p} C$ module, hence $\operatorname{res}_{C}^{G}\left\{H^{2}\left(G, \mathbf{F}_{p}\left[G / H_{i}\right]\right)\right\}=0$. So, $C$ must be a subgroup of $H_{i}$. We can write the map $\psi_{i}$ as the composition

$$
\begin{aligned}
\psi_{i}: H^{2}\left(G, \mathbf{F}_{p}\left[G / H_{i}\right]\right) & \stackrel{\operatorname{res}_{H_{i}}^{G}}{\longrightarrow} H^{2}\left(H_{i}, \operatorname{res}_{H_{i}}^{G} \mathbf{F}_{p}\left[G / H_{i}\right]\right) \\
& =H^{2}\left(H_{i}, \bigoplus_{g \in G / H_{i}} g \mathbf{F}_{p}\right) \longrightarrow H^{2}\left(H_{i}, \mathbf{F}_{p}\right)
\end{aligned}
$$

where the second map is the projection $\underset{g \in G / H_{i}}{\bigoplus_{p}} g \mathbf{F}_{p} \rightarrow g_{0} \mathbf{F}_{p} \cong \mathbf{F}_{p}$ where $g_{0}$ is the coset representative for the identity element of $G / H_{i}$. Observe that $G$ acts on the middle term by permuting the summands, and the image of $r e s_{H_{i}}^{G}$ is invariant under this action. This implies that $\operatorname{res}_{K}^{G} \psi(u)=0$ if and only if $\operatorname{res}_{K}^{G} u=0$ for every subgroup $K \subseteq H_{i}$ and every element $u \in H^{2}\left(G, \mathbf{F}_{p}\left[G / H_{i}\right]\right)$. So, $\operatorname{res}_{C}^{G} \psi\left(\alpha_{i}\right)$ must be nonzero. Since $\pi_{i}$ reduces to the identity homomorphism on subgroups of $H_{i}, \pi_{i}^{*}$ reduced to $C$ is injective. Hence

$$
\operatorname{res}_{C}^{G} \phi\left(\alpha_{i}\right)=\left.\pi_{i}^{*}\right|_{C} \circ \operatorname{res}_{C}^{G} \psi_{i}\left(\alpha_{i}\right) \neq 0 .
$$

So, $\theta$ is a special class.

Now, we will show that $\theta$ is an integral class, i.e. an image of a class in $H^{2}\left(G, \mathbf{Z}^{l}\right)$. For this, it is enough to show that its image under the Bockstein homomorphism, $\beta$ is zero. Since $\beta(\alpha)=0, \beta(\theta)$ will be zero if $\phi_{i}$ commutes with $\beta$. This is clear since all the maps involved in the composition commute with $\beta$.

Let $\bar{\theta} \in H^{2}\left(G, \mathbf{Z}^{l}\right)$ be a preimage of $\theta$ under the $\bmod p$ reduction map. Since $\theta$ is special, $\bar{\theta}$ must be special also. Hence, it represents an extension $0 \rightarrow \mathbf{Z}^{l} \rightarrow$ $\Gamma^{\prime} \rightarrow G \rightarrow 1$ with $\Gamma^{\prime}$ torsion free. Applying Theorem 3.2, we conclude that $r \leq l=$ $\operatorname{dim}_{\mathbf{F}_{p}}\left(\mathbf{F}_{p} \otimes M\right)^{G}$.

\section{Group actions on a torus}

In this section we link the results of the previous sections to group actions. Let $X$ be a finite dimensional manifold or a finite dimensional $C W$ complex homotopy equivalent to $\left(S^{1}\right)^{k}$ for some $k$, which we abbreviate as $X \simeq\left(S^{1}\right)^{k}$. Let $G$ be an arbitrary finite group which acts freely on $X$. We will assume that the action is a smooth action on a manifold or a cellular action on a $C W$ complex. This 
assumption guarantees that the orbit space $X / G$ is also finite dimensional. This is one of the main topological inputs we have.

Since the orbit map $q: X \rightarrow X / G$ is a regular covering map, we get a short exact sequence of groups

$$
0 \rightarrow \pi_{1}\left(X, x_{0}\right) \rightarrow \pi_{1}\left(X / G, \bar{x}_{0}\right) \rightarrow G \rightarrow 1
$$

Note that there is a $G$ action on $\pi_{1}\left(X, x_{0}\right)$, coming from the topological action on $X$. Such an action exists because $\pi_{1}\left(X, x_{0}\right)$ is abelian. We will consider $\pi_{1}\left(X, x_{0}\right)$ as a $\mathbf{Z} G$-module with this action, and we denote it by $M$. Since the action induced from the topological action coincides with the conjugation action in $\pi_{1}\left(X / G, \bar{x}_{0}\right)$, the above extension is an extension for the $\mathbf{Z} G$-module $M$. Let $\Gamma=\pi_{1}\left(X / G, \bar{x}_{0}\right)$. We have the following:

Lemma 5.1. $\Gamma$ is torsion free.

Proof. Since $\pi_{i}\left(X / G, \bar{x}_{0}\right)=\pi_{i}\left(X, x_{0}\right)=0$ for $i>1, X / G$ is a classifying space for $\Gamma$. Note that $X / G$ is finite dimensional because $X$ is finite dimensional. Hence $H^{*}(\Gamma, \mathbf{Z})=H^{*}(X / G, \mathbf{Z})$ is finite dimensional. Now, assume that $\Gamma$ is not torsion free. Then $\mathbf{Z} / n \hookrightarrow \Gamma$ for some $n$, and we can consider $H^{*}(\mathbf{Z} / n, \mathbf{Z})$ as a $H^{*}(\Gamma, \mathbf{Z})$ module through the restriction map. By a result of L. Evens 10 , $H^{*}(\mathbf{Z} / n, \mathbf{Z})$ is finitely generated over $H^{*}(\Gamma, \mathbf{Z})$. Since $H^{*}(\Gamma, \mathbf{Z})$ is finite dimensional, this will imply that $H^{*}(\mathbf{Z} / n, \mathbf{Z})$ is finite dimensional. This contradicts the known computation $H^{2 i}(\mathbf{Z} / n, \mathbf{Z})=\mathbf{Z} / n$ for all $i>0$.

Combining this with the results of previous sections we obtain:

Corollary 5.2. If $G=(\mathbf{Z} / 2)^{r}$ acts freely on $X \simeq\left(S^{1}\right)^{k}$, then $r \leq k$.

Proof. This follows immediately from Lemma 5.1] and Theorem 3.2.

We also recover the $n=1$ case of Adem and Benson's theorem [2].

Corollary 5.3 (Adem and Benson). If $G=(\mathbf{Z} / p)^{r}$ acts freely on $X \simeq\left(S^{1}\right)^{k}$, permuting a basis for $H_{1}\left(X, \mathbf{F}_{p}\right)$, then

$$
r \leq \operatorname{dim}_{\mathbf{F}_{p}} H_{1}\left(X, \mathbf{F}_{p}\right)^{G} .
$$

Proof. This immediately follows from Lemma 5.1 and Proposition 4.3.

Now, we consider the case where $G$ is an arbitrary finite group and the action on homology is trivial. We prove the following:

Theorem 5.4. If a finite group $G$ acts freely on $X \simeq\left(S^{1}\right)^{k}$ with trivial action on homology, then $G$ is abelian.

Proof. Let $M$ and $\Gamma$ be as above. Since the action on homology is trivial, we have $M=M^{G} \subseteq Z(\Gamma)$. Hence

$$
|\Gamma / Z(\Gamma)| \leq|\Gamma / M|=|G|<\infty .
$$

Now, we quote a result from group theory:

Lemma 5.5 (Schur). If $\Gamma$ is a group with $\Gamma / Z(\Gamma)$ finite, then $\Gamma^{\prime}$ is also finite.

Proof. See page 114 in [14].

From this, it follows that $\Gamma^{\prime}$ is finite. However, by Lemma 5.1, $\Gamma$ is torsion free. Hence, $\Gamma^{\prime}$ has to be the trivial, i.e. $\Gamma$ is abelian. Therefore $G$ is abelian. 
Recently it was brought to my attention that Theorem 5.4 can be obtained as a special case of a result in [11].

\section{EXTENSIONS BY ELEMENTARY ABELIAN P-GROUPS}

Throughout this section we will consider central extensions of the form

$$
0 \rightarrow E \rightarrow Q \rightarrow G \rightarrow 1
$$

where $E=(\mathbf{Z} / p)^{k}$ and $G=(\mathbf{Z} / p)^{r}$.

Observe that extension (3) is special if and only if $E$ is a maximal elementary abelian subgroup of $Q$. In this case $Q$ would satisfy the $p C$ condition, i.e. every element of order $p$ will be central.

Lemma 6.1. Suppose extension (3) is special, then

$$
r \leq \begin{cases}2 k & \text { if } p=2, \\ k & \text { if } p>2 .\end{cases}
$$

Proof. This lemma was proved for $p=2$ by Cusick [9] and for $p>2$ by Allday [3]. For completeness, we include the proof of the $p=2$ case. Let $\alpha \in H^{2}(G, E)$ be the extension class for (3). Since $E=(\mathbf{Z} / 2)^{k}$ is a trivial $G$-module, $H^{2}(G, E) \cong$ $\bigoplus_{i=1}^{k} H^{2}(G, \mathbf{Z} / 2)$. Hence one can write $\alpha=\left(\alpha_{1}, \ldots, \alpha_{k}\right)$ where $\alpha_{i} \in H^{2}(G, \mathbf{Z} / 2)$. Since $H^{*}(G, \mathbf{Z} / 2) \cong \mathbf{Z} / 2\left[x_{1}, \ldots, x_{r}\right]$ with $\operatorname{deg} x_{i}=1$, we can consider $\alpha_{i}$ 's as quadratic polynomials in variables $x_{1}, \ldots, x_{r}$. By a well known argument of Carlsson, $\alpha$ being special implies that $\alpha_{1}, \ldots, \alpha_{k}$ do not have a common zero 7]. Hence $r \leq 2 k$ follows from the proposition.

Proposition 6.2 (Lang-Nagata). If $f_{1}, \ldots, f_{k}$ are homogeneous polynomials in $r$ variables of degree $n$ with coefficients in $\mathbf{Z} / 2$, and if $r>n k$, then they have $a$ nontrivial common zero in $(\mathbf{Z} / 2)^{r}$.

Proof. See page 18 of [12].

Now, we will prove a refinement of Lemma 6.1 for $p=2$.

Proposition 6.3. Let $0 \rightarrow E \rightarrow Q \rightarrow G \rightarrow 1$ be a special central extension with $E=(\mathbf{Z} / 2)^{k}, G=(\mathbf{Z} / 2)^{r}$. Then $r \leq k+\operatorname{rk} Q^{\prime}$.

Proof. Consider the following diagram where all the boxes commute.

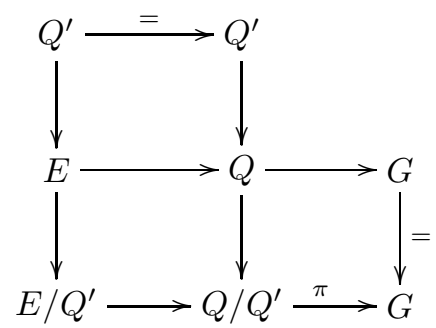

Let $\psi: G \rightarrow E / Q^{\prime}$ be the $2^{\text {nd }}$ power map defined as $\psi(g)=(\hat{g})^{2}$ where $\hat{g} \in Q / Q^{\prime}$ is the coset representative for $g \in G$. Since $Q / Q^{\prime}$ is abelian, $\phi$ is a homomorphism. Let $K=\operatorname{ker} \psi$. Then we have

$$
\operatorname{rk}(G / K) \leq \operatorname{rk}\left(E / Q^{\prime}\right)
$$


Now, consider

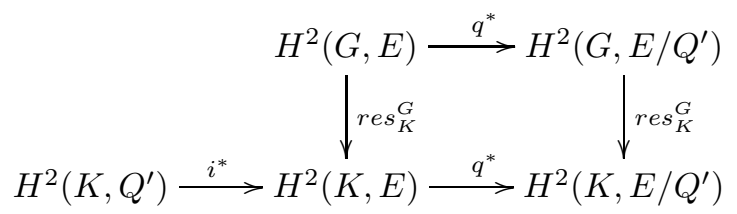

where rows of the diagram come from the short exact sequence

$$
0 \rightarrow Q^{\prime} \longrightarrow E \stackrel{q}{\longrightarrow} E / Q^{\prime} \rightarrow 1
$$

If $\alpha \in H^{2}(G, E)$ is the extension class for $\quad 0 \rightarrow E \rightarrow Q \rightarrow G \rightarrow 1$, then $\operatorname{res}_{K}^{G} q^{*}(\alpha)$ represents the extension $0 \rightarrow E / Q^{\prime} \rightarrow \pi^{-1}(K) \rightarrow K \rightarrow 1$. Since $\psi(k)=(\hat{k})^{2}=1$ for each $k \in K$, this extension splits, hence $\operatorname{res}_{K}^{G} q^{*}(\alpha)=0$.

From the commutativity of the above cohomology diagram, we get $q^{*}\left(\operatorname{res}_{K}^{G} \alpha\right)=$ 0 . Hence there exists an element $\alpha^{\prime} \in H^{2}\left(K, Q^{\prime}\right)$ such that $i^{*}\left(\alpha^{\prime}\right)=r e s_{K}^{G} \alpha$. Now, $\alpha^{\prime}$ must be special, because otherwise, there exists a cyclic subgroup $C$ of $K$ such that $\operatorname{res}_{C}^{K} \alpha^{\prime}=0$, giving

$$
\operatorname{res}_{C}^{G} \alpha=\operatorname{res}_{C}^{K} \operatorname{res}_{K}^{G} \alpha=\operatorname{res}_{C}^{K} i^{*}\left(\alpha^{\prime}\right)=i^{*}\left(\operatorname{res}_{C}^{K} \alpha^{\prime}\right)=0
$$

which is a contradiction to the speciality of $\alpha$.

Finally, we apply Lemma 6.1 to the extension represented by $\alpha^{\prime}$ and we get

$$
\operatorname{rk} K \leq 2 \operatorname{rk} Q^{\prime}
$$

which, together with (4), gives the desired inequality.

We conclude this section with an application to group theory. Let $r_{2}(G)$ denote the 2-rank of a group $G$, the largest integer $r$ such that $G$ has a subgroup isomorphic to $(\mathbf{Z} / p)^{r}$.

Corollary 6.4. Let $G$ be a 2-group satisfying the $2 C$ condition and let $E \subseteq G$ be the elementary abelian subgroup of maximum rank. Then

$$
r_{2}(G / E) \leq r_{2}(G)+r_{2}\left(G^{\prime}\right) .
$$

\section{Commutator subgroup of a Class 2 Group}

Let $G$ be a finite, class 2 group, i.e. $G$ has a central subgroup $H$ with an abelian quotient. Consider the following diagram

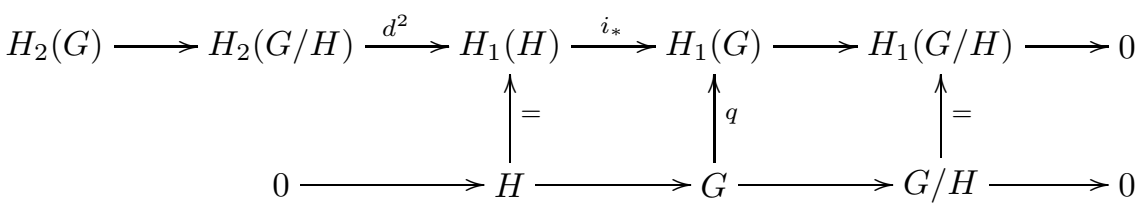

where the first row is the homology 5-term exact sequence of the Hochschild-Serre spectral sequence in $\mathbf{Z}$ coefficients. Note also that the boxes in the diagram are commutative where the horizontal map $q$ is the abelianization map. From this we get

$$
\operatorname{im} d^{2}=\operatorname{ker} i_{*}=(\operatorname{ker} q) \cap H=G^{\prime} \cap H=G^{\prime} .
$$

Now, consider the cohomology 5 -term exact sequence for the same extension

$$
0 \rightarrow H^{2}(G / H) \rightarrow H^{2}(G) \rightarrow H^{2}(H) \stackrel{d_{3}}{\longrightarrow} H^{3}(G / H) \rightarrow H^{3}(G)
$$


which gives a short exact sequence of finite groups

$$
0 \rightarrow H^{2}(G / H) \rightarrow H^{2}(G) \rightarrow \operatorname{ker} d_{3} \rightarrow 0 .
$$

We have the following duality:

Lemma 7.1. $\operatorname{Ext}\left(\operatorname{ker} d_{3}, \mathbf{Z}\right) \cong H / \operatorname{im} d^{2}$.

Proof. Consider the diagram

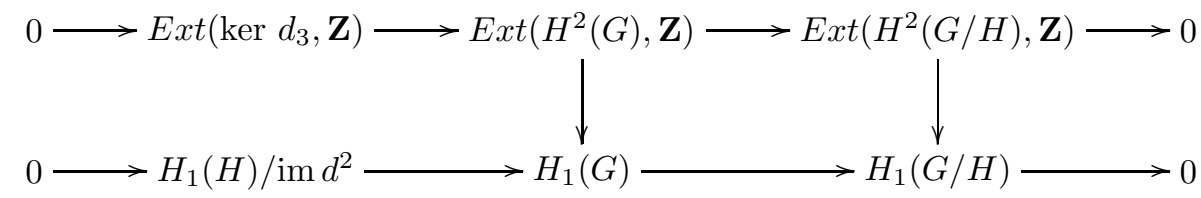

where the first row comes from the short exact sequence (15), and the second row comes from the 5 -term exact sequence for homology. Since $G$ is a finite group, both vertical maps are isomorphisms by the Universal Coefficient Theorem. Hence, the induced map

$$
\operatorname{Ext}\left(\operatorname{ker} d_{3}, \mathbf{Z}\right) \longrightarrow H / \operatorname{im} d^{2}
$$

is an isomorphism.

Proposition 7.2. Let $G$ be a finite, class 2 group. Let $H \subseteq G$ be a central subgroup such that $G / H$ is abelian. Then

$$
G^{\prime} \cong \operatorname{im}\left\{d_{3}: H^{2}(H) \rightarrow H^{3}(G / H)\right\} .
$$

Proof. We have $H / G^{\prime}=H / \operatorname{im} d^{2} \cong \operatorname{Ext}\left(\operatorname{ker} d_{3}, \mathbf{Z}\right) \cong \operatorname{ker} d_{3}$ because ker $d_{3}$ is a finite abelian group. Hence $G^{\prime} \cong H / \operatorname{ker} d_{3} \cong \operatorname{im} d_{3}$.

As a consequence one immediately gets that if $G / H=(Z / p)^{r}$, then $\left|G^{\prime}\right| \leq p^{\frac{r(r-1)}{2}}$. In the next section we give a topological application.

\section{Actions on products of ReAl projective spaces}

Let $X$ be a finite $C W$ complex, homotopy equivalent to a product of odd dimensional real projective spaces, which we abbreviate as $X \simeq \prod_{i=1}^{k} \mathbf{R} P^{2 m_{i}+1}$.

Lemma 8.1. Let $G=(\mathbf{Z} / 2)^{r}$, and let $X \simeq \prod_{i=1}^{k} \mathbf{R} P^{2 m_{i}+1}\left(m_{i}>0\right.$ for all $\left.i\right)$ be $a$ free $G$-space with a trivial action on mod 2 cohomology. If $n$ is the number of odd $m_{i}$ 's, then

$$
\operatorname{rk} \pi_{1}\left(X / G, \bar{x}_{0}\right)^{\prime} \leq n .
$$

Proof. Consider the Cartan-Leray spectral sequence

$$
H^{*}\left(G, H^{*}(X, \mathbf{Z})\right) \Rightarrow H^{*}(X / G, \mathbf{Z})
$$

associated to the regular covering map $X \rightarrow X / G$. Let $\left\{x_{1}, \ldots, x_{k}\right\}$ be a generating set for $H^{2}(X, \mathbf{Z})=H^{2}\left(\prod_{i=1}^{k} \mathbf{R} P^{2 m_{i}+1}, \mathbf{Z}\right)$ where $x_{i}$ corresponds to $\mathbf{R} P^{2 m_{i}+1}$. If $m_{i}=2 l$ for some $l$, then by using the derivation property of the differentials, we get

$$
0=d_{3}\left(x_{i}^{2 l+1}\right)=x_{i}^{2 l} \otimes d_{3}\left(x_{i}\right)
$$


which gives that $d_{3}\left(x_{i}\right)=0$. Hence, $\operatorname{dim}_{\mathbf{F}_{p}}\left(\operatorname{ker} d_{3}\right)$ is greater or equal to the number of even $m_{i}$ 's, i.e. $\operatorname{dim}_{\mathbf{F}_{p}}\left(\operatorname{ker} d_{3}\right) \geq k-n$.

Now, note that $d_{3}$ in the above C-L spectral sequence is the same as $d_{3}$ of the $\mathrm{H}-\mathrm{S}$ spectral sequence for

$$
0 \rightarrow \pi_{1}\left(X, x_{0}\right) \rightarrow \pi_{1}\left(X / G, \bar{x}_{0}\right) \rightarrow G \rightarrow 1
$$

and for the latter $d_{3}$, we have

$$
\operatorname{rk} \pi_{1}\left(X / G, \bar{x}_{0}\right)^{\prime}=k-\operatorname{dim}_{\mathbf{F}_{p}}\left(\operatorname{ker} d_{3}\right)
$$

from Proposition 7.2. Combining this with the above inequality gives the desired inequality.

Lemma 8.2. Let $G=(\mathbf{Z} / 2)^{r}$ and let $X$ be a finite $C W$ complex such that $\pi_{1}\left(X, x_{0}\right)$ is abelian and $H^{*}\left(X, \mathbf{F}_{2}\right)$ is generated by one-dimensional generators. If $G$ acts freely on $X$ with a trivial action on homology, then the homotopy sequence

$$
0 \rightarrow \pi_{1}\left(X, x_{0}\right) \rightarrow \pi_{1}\left(X / G, \bar{x}_{0}\right) \rightarrow G \rightarrow 1
$$

is a special extension.

Proof. Let $\alpha$ be the extension class for this extension. Then for a cyclic subgroup $C \subseteq G, \operatorname{res}_{C}^{G} \alpha$ will be the extension class for the homotopy sequence

$$
0 \rightarrow \pi_{1}\left(X, x_{0}\right) \longrightarrow \pi_{1}\left(X / C, \bar{x}_{0}\right) \stackrel{q}{\longrightarrow} C \rightarrow 1
$$

associated to the reduced $C$ action on $X$.

Assume that $\operatorname{res}_{C}^{G} \alpha=0$ for some $C \subseteq G$, i.e. $q: \pi_{1}\left(X / C, \bar{x}_{0}\right) \rightarrow C$ splits. Let $s$ be a splitting map for $q$, then the composition

$$
H_{1}(C, \mathbf{Z})=C \stackrel{s}{\longrightarrow} \pi_{1}\left(X / C, \bar{x}_{0}\right) \stackrel{a b}{\longrightarrow} H_{1}(X / C, \mathbf{Z})
$$

gives a splitting for homology. Hence

$$
H_{1}\left(X / C, \mathbf{F}_{2}\right) \cong H_{1}\left(X, \mathbf{F}_{2}\right) \oplus H_{1}\left(C, \mathbf{F}_{2}\right)
$$

whose dual gives that $i^{*}: H^{1}\left(X / C, \mathbf{F}_{2}\right) \rightarrow H^{1}\left(X, \mathbf{F}_{2}\right)$ is a surjection.

Now, consider the Cartan-Leray spectral sequence

$$
H^{p}\left(C, H^{q}\left(X, \mathbf{F}_{2}\right)\right) \Rightarrow H^{p+q}\left(X / C, \mathbf{F}_{2}\right)
$$

associated to the covering $X \rightarrow X / C$. Let

$$
d_{2}^{p, q}: H^{p}\left(C, H^{q}\left(X, \mathbf{F}_{2}\right)\right) \rightarrow H^{p+2}\left(C, H^{q-1}\left(X, \mathbf{F}_{2}\right)\right)
$$

denote the differentials in $E_{2}$. Note that for $(p, q)=(0,1)$, we have $\operatorname{ker} d_{2}^{0,1}=\operatorname{im} i^{*}$. So, the surjectivity of $i^{*}$ gives that $d_{2}^{0,1}$ is identically zero. Then, $d_{2}^{p, q}=0$ for all $p, q \geq 0$ because $H^{*}\left(X, \mathbf{F}_{2}\right)$ is generated by $H^{1}\left(X, \mathbf{F}_{2}\right)$. Since higher differentials $d_{r}^{0,1}$ are also zero, the spectral sequence collapses, i.e.

$$
H^{*}\left(X / C, \mathbf{F}_{2}\right) \cong H^{*}\left(X, \mathbf{F}_{2}\right) \otimes H^{*}\left(C, \mathbf{F}_{2}\right) .
$$

This gives that $H^{*}\left(X / C, \mathbf{F}_{2}\right)$ is infinite dimensional. On the other hand, $X / C$ is a finite $C W$ complex, hence $H^{*}\left(X / C, \mathbf{F}_{2}\right)$ must be finite dimensional. We reached to a contradiction, hence the proof is complete.

Now, we state the main result of this section. 
Theorem 8.3. If $G=(\mathbf{Z} / 2)^{r}$ acts freely and mod 2 acts homologically trivially on a finite complex $X \simeq \prod_{i=1}^{k} \mathbf{R} P^{2 m_{i}+1}$ where $m_{i}>0$ for all $i$, then $r \leq \mu\left(m_{1}\right)+\cdots+$ $\mu\left(m_{k}\right)$, where $\mu\left(m_{i}\right)=1$ for $m_{i}$ even and $\mu\left(m_{i}\right)=2$ for $m_{i}$ odd.

Proof. By Lemma 8.2 , central extension

$$
0 \rightarrow \pi_{1}\left(X, x_{0}\right) \rightarrow \pi_{1}\left(X / G, \bar{x}_{0}\right) \rightarrow G \rightarrow 1
$$

is a special extension. Applying Proposition 6.3 we get that $r \leq k+\pi_{1}\left(X / G, \bar{x}_{0}\right)^{\prime}$. Combining this with the inequality in Lemma 8.1 we obtain the desired inequality.

We conclude this paper with an application of Lemma 8.2, suggested by A. Adem.

Corollary 8.4. Suppose that $G=(\mathbf{Z} / 2)^{r}$ acts freely on $X=S^{n_{1}} \times \cdots \times S^{n_{k}}$, and suppose further that for some subgroup $H \subseteq G$ of rank $k, H^{*}\left(X / H, \mathbf{F}_{2}\right)$ is generated by one-dimensional classes. Then $r=k$.

\section{REFERENCES}

[1] A. Adem and D.J. Benson, Abelian groups acting on products of spheres, Math. Z. 228 (1998), 705-712. CMP 99:01

[2] A. Adem and W. Browder, The free rank of symmetry on $\left(S^{n}\right)^{k}$, Invent. Math. 92 (1988), 431-440. MR 89e:57034

[3] C. Allday, Elementary abelian p-group actions on lens spaces, Topology Hawaii (Honolulu, HI, 1990), 1-11, World Sci. Publishing, River Edge, NJ, 1992. MR 93e:57068

[4] D.J. Benson and J.F. Carlson, Complexity and multiple complexes, Math. Zeit. 195 (1987), 221-238. MR 88e:20050

[5] W. Browder, Cohomology and group actions, Invent. Math. 71 (1983), 599-607. MR 85m:57022a

[6] K.S. Brown, Cohomology of groups, Springer-Verlag Graduate Texts in Math 87, 1994. MR 96a:20072

[7] G. Carlsson, On the non-existence of free actions of elementary abelian groups on products of spheres, Amer. J. Math. 102 (1980), 1147-1157. MR 82a:57038

[8] G. Carlsson, On the rank of abelian groups acting freely on $\left(S^{n}\right)^{k}$, Invent. Math. 69 (1982), 393-400. MR 84e:57033

[9] L.W. Cusick, Elementary abelian 2-groups that can act freely on products of real projective spaces, Proc. Amer. Math. Soc. 87 (1983), 728-730. MR 84f:57024

[10] L. Evens, The cohomology ring of a finite group, Trans. Amer. Math. Soc. 101 (1961), 224-239. MR 25:1191

[11] D.H. Gottlieb, K.B. Lee, and M. Özaydın, Compact group actions and maps into $K(\pi, 1)$ spaces, Trans. Amer. Math. Soc. 287 (1985), 419-429. MR 86h:57034

[12] M. Greenberg, Lectures on forms in many variables, Benjamin, New York, 1969. MR 39:2698

[13] H. Hiller, Z. Marciniak, C.-H. Sah, and A. Szczepanski, Holonomy of flat manifolds with $b_{1}=0$. II , Quart. J. Math. 38 (1987), 213-220. MR 88f:53074

[14] J.J. Rotman, An Introduction to the theory of groups, Springer-Verlag Graduate Texts in Math 148, 1995. MR 95m:20001

Department of Mathematics, University of Wisconsin, Madison, Wisconsin 53706

Current address: Department of Mathematics, Indiana University, Bloomington, Indiana 47405

E-mail address: eyalcin@math.indiana.edu 